\title{
Impact of a Prenatal Cognitive-Behavioral Stress Management Intervention on Salivary Cortisol Levels in Low-Income Mothers and their Infants
}

\author{
Guido G. Urizar Jr., Ph.D. and \\ Department of Psychology, California State University, Long Beach \\ Ricardo F. Muñoz, Ph.D. \\ Department of Psychiatry, University of California, San Francisco
}

\begin{abstract}
Recent findings suggest that elevated stress levels during the pre- and postpartum period are related to poor maternal and infant health outcomes; yet, few studies have prospectively examined the efficacy of stress management interventions on regulating stress levels among mothers and their infants. The current study examined whether a prenatal cognitive behavioral stress management (CBSM) intervention would be effective in regulating salivary cortisol (a biological marker of stress) and self-reported stress levels among mothers and their infants at six and 18 months postpartum, relative to two control groups. Our sample was comprised of predominantly Spanish-speaking, low-income women $(80 \%$; mean age $=25 \pm 5$ years $)$ who were screened for depression during their second trimester of pregnancy ( $M=16 \pm 5$ weeks of gestation). Women at high risk for depression [i.e., having either a past history of major depression or current elevated symptoms of depression ( $\geq 16$ on CES-D)] were randomized to either a CBSM group $(n=24)$ or a usual care (UC) group $(n=33)$, while a low risk comparison (LRC) group $(n=29)$ was comprised of women not meeting either depression criteria. ANCOVA analyses demonstrated that: 1) infants of women in the CBSM and LRC groups had significantly lower cortisol levels than infants of women in the UC group at six months postpartum ( $<.001)$; and 2) women in the CBSM group had lower cortisol levels than women in the UC group at 18 months postpartum $(\mathrm{p}<.01)$. These results suggest that prenatal CBSM interventions may be efficacious in regulating biological markers of stress among mothers and their infants, thereby decreasing their risk for developing health complications over time.
\end{abstract}

\section{Keywords}

stress; cortisol; pregnancy; mothers; infants; stress management

\begin{abstract}
Although stress is generally considered to be an adaptive response to challenges in the environment, stressors occurring during pregnancy may negatively affect the health of expectant mothers and their infants. Prenatal stress has been implicated as a trigger for several physical and mental health problems for women, including poor nutrition, increased cardiovascular disease risk, increased anxiety, and postpartum depression (Da Costa,
\end{abstract}

Send Correspondence to: Guido G. Urizar, Jr., Ph.D., Department of Psychology, California State University, Long Beach, 1250 Bellflower Blvd, Long Beach, CA 90714-5109, Phone: (562) 985-5160, Fax: (562) 985-8004, gurizar@csulb.edu.

Publisher's Disclaimer: This is a PDF file of an unedited manuscript that has been accepted for publication. As a service to our customers we are providing this early version of the manuscript. The manuscript will undergo copyediting, typesetting, and review of the resulting proof before it is published in its final citable form. Please note that during the production process errors may be discovered which could affect the content, and all legal disclaimers that apply to the journal pertain. 
Larouche, Drista, \& Brender, 2000; Lobel et al., 2008). Prenatal stress has also been linked to adverse birth outcomes, such as preterm births and low birthweight, which are the leading causes of mortality and morbidity among newborns and are associated with significantly higher rates of neurodevelopmental impairments (Knoches \& Doyle, 1993). Prenatal stress could therefore have long-term consequences for both the mother and her child.

The stress hormone cortisol has been identified as a possible biological mechanism associated with the heightened risk for developing health complications during pregnancy and the postpartum period. Cortisol is a hormone that is modulated by the hypothalamicpituitary-adrenal (HPA) axis, is released by the adrenal glands in response to stressors in our environment, and is considered one of the main markers associated with the biological stress response (Austin \& Leader, 2000). An abrupt and dramatic increase in cortisol occurs during pregnancy and the early postpartum period, with biologically active (free) cortisol levels rising after the $25^{\text {th }}$ week of gestation and returning to normal approximately 15 days postpartum (Hendrick, Altshuler, \& Suri, 1998). A diurnal cortisol pattern is still observed during pregnancy, with salivary cortisol levels being highest in the morning and lowest at the end of the day (Harris, 1996). Although the rise in cortisol during pregnancy is a normal occurrence, some studies have demonstrated that higher elevations in prenatal salivary cortisol are related to several adverse birth outcomes, including increased rates of pre- and post-mature births, a higher need for infant resuscitation assistance at birth (McCool et al., 1994; Ponirakis et al., 1997), and infant brain cell damage, which can lead to long-term cognitive and emotional deficits later in childhood (Gunnar, 1998; Kurstjens \& Wolke, 2001).

These prenatal changes in maternal biochemistry are highly significant in fetal development, as maternal cortisol readily crosses the placenta during the first and second trimesters (Gitau et al., 1998; Goodman \& Gotlib, 1999). In fact, infants of mothers who exhibit elevated cortisol levels during pregnancy have been shown to have high cortisol levels after birth, thus mimicking their mother's prenatal cortisol levels (Diego et al., 2004; Field et al., 2004; Lundy et al., 1999). Salivary cortisol levels have also been shown to be higher among infants of mothers who experience chronic stress and depression during the early postpartum period, leading to the later development of behavioral, mental health, and cognitive deficits in preschool and elementary-school children (Ashman, Dawson, Panagiotides, Yamada, \& Wilkinson, 2002; Essex, Klein, Cho, \& Kalin, 2002; Kurstjens \& Wolke, 2001). Collectively, these findings suggest that elevated cortisol levels during pregnancy and the early postpartum period negatively affect mothers and their infants. Furthermore, these findings highlight the need to develop and test prenatal interventions that target optimal management of stress levels during this critical period of human development.

Several recent studies have shown cognitive-behavioral stress management (CBSM) interventions to be effective in reducing both salivary and plasma cortisol levels in healthy adults without any medical complications (Gaab et al., 2003) and patients with stressful medical conditions, such as HIV and breast cancer (Antoni, 2003; Cruess et al., 2000). The components of these CBSM interventions have largely consisted of standard cognitive behavioral strategies and relaxation techniques that are used to decrease symptoms of depression and anxiety in patients with mental health disorders and include, but are not limited to, cognitive restructuring, problem-solving, progressive muscle relaxation, and guided imagery (Barlow \& Cerny, 1988; Beck, 1991; Cruess et al., 2000; Gaab et al., 2003).

Although CBSM interventions have been found to be effective in regulating cortisol levels in non-pregnant populations, few studies have systematically evaluated their effectiveness in regulating cortisol during pregnancy and the postpartum period. One study examined the effects of providing pregnant women with brief instructions to have a day of stress reduction 
on their salivary cortisol and stress levels, which were assessed prior to and after receiving these instructions (Urizar et al., 2004). More specifically, women were asked to eliminate things that were stressful to them and/or participate in things that would increase their level of relaxation. Compared to their baseline levels, women demonstrated significant decreases in both prenatal cortisol and stress after having received these instructions. Interestingly, the majority of women in this study reported engaging in pleasant activities (e.g., walking outside), seeking out positive interactions with others, and modifying their thoughts - key cognitive behavioral strategies for managing stress and depression (Muñoz \& Ying, 1993) in order to reduce their stress levels during pregnancy. Similar findings for stress and both urinary and salivary cortisol have been reported for pregnant women receiving massage therapy (Field, Diego, Hernandez-Reif, Schanberg, \& Kuhn, 2004) and those taking part in community-based stress management programs (Wesley, 2006). Although these results provide some support for the use of CBSM in regulating cortisol levels among pregnant women, these studies are limited in that they either did not include a control group or had a short follow-up period.

A second study randomly assigned depressed adolescent mothers to either a 12-week postpartum lifestyle intervention - which included a rehabilitation program (social/ educational/vocational), mood induction activities (i.e., relaxation, massage, and music therapy), and mother-infant interaction coaching - or a control condition shortly after childbirth (Field et al., 2000). Results demonstrated that mothers who received the lifestyle intervention had significantly lower urinary cortisol levels (as well as more positive interactions with their infants) at six months postpartum, relative to mothers in the control condition. Although these results may be useful in identifying intervention strategies that help manage stress during the postpartum period, the comprehensive nature of this lifestyle intervention makes it difficult to determine which intervention components are critical in achieving successful cortisol and stress regulation in this population. Nevertheless, these results highlight the need to prospectively examine the efficacy of prenatal CBSM interventions in producing short- and long-term reductions in cortisol and stress among mothers and their infants.

The current study focused on a subset of participants from the Mothers and Babies: Mood and Health Project, which was a pilot study designed to prevent the onset of major depressive episodes among low-income, pregnant women who were identified as being at high risk for developing depression (Muñoz et al., 2007a). Diurnal salivary cortisol and stress ratings were collected from these women and their infants to determine whether their participation in a prenatal CBSM intervention would result in lower cortisol and selfreported stress levels, relative to two control groups (i.e., usual care group and a low risk comparison group). Women who were identified to be at high risk for depression during pregnancy were randomized to either a CBSM intervention or a usual care (UC) group, while women identified to be at low risk for depression took part in a low risk comparison (LRC) group. It was hypothesized that women receiving the CBSM intervention would have lower cortisol and self-reported stress levels during the postpartum period than UC women, and have similar cortisol and stress levels as women in the LRC group. In addition, infants of mothers receiving the CBSM intervention were expected to have lower cortisol levels than infants of mothers in UC, and have similar cortisol levels as infants of mothers in the LRC group.

\section{Method}

\section{Participants and Procedure}

The objective of the Mothers and Babies: Mood and Health Project was to reduce the incidence of major depression in low-income, pregnant women receiving prenatal health 
services in a public sector hospital in California (Muñoz et al., 2007a; Urizar et al., 2004). Of the 110 pregnant women who participated in the original study, the current results focus on the 86 women and their infants $(78 \%)$ for whom cortisol samples were collected from 2000 to 2003.

Women were eligible to join the study if they were 18 years of age or older, six to 28 weeks pregnant (this gestational age range was used to ensure that women would have enough time to complete the 12-week, prenatal CBSM intervention and to facilitate recruitment into the Mothers and Babes: Mood and Health Project), fluent in either Spanish or English, and free of any major medical or substance abuse problems (e.g., high risk pregnancy status). Women were assessed to determine whether they were receiving treatment with steroid or antidepressant medications, given that these could affect cortisol levels. No women were receiving these medications at baseline or throughout the study. Eligibility criteria were assessed through an initial medical record review. Women were then approached in the waiting room during their prenatal clinic visits, given brochures or flyers, or were provided with information about the study by their health care providers. After consent was obtained, eligible women participated in a screening interview that assessed their depression risk status. The screening interview consisted of assessing women for a past history of major depressive episodes (MDEs) and current depressive symptoms (using the Maternal Mood Screener and CES-D, respectively; described below). At baseline (i.e., 6-28 weeks of gestation), women identified to be at high risk for depression (i.e., having a past history of a MDE and/or $\geq 16$ on the CES-D; see Le et al., 2004) were assessed for salivary cortisol and self-reported stress levels and subsequently randomized to either a CBSM or UC group. They were then reassessed at six and 18 months postpartum. Women who were at low risk for depression (i.e., no past history of MDEs and $<16$ on the CES-D) were part of a LRC group and invited to participate in a separate observational study that assessed for salivary cortisol and stress levels during pregnancy and at six months postpartum (Urizar et al., 2004). Finally, women who were currently depressed (i.e., currently experiencing five of nine MDE symptoms for at least a two-week period, per DSM-IV criteria; American Psychiatric Association, 1994) were excluded from the original study and referred for a formal clinical evaluation. No women were excluded based on their baseline stress levels. All study procedures were approved by the Institutional Review Board at the University of California, San Francisco.

\section{Research Design}

Upon their entry into the study at baseline (i.e., 6-28 weeks of gestation), eligible participants took part in a 30-minute information session where the procedures for collecting salivary cortisol samples at home and completing self-report questionnaires were explained by a research assistant at the hospital where they received prenatal care. There was an approximate window of two weeks between the time participants completed their baseline assessments and the initiation of their randomization group. Participants were instructed to collect two salivary cortisol samples (morning and evening) on one collection day prior to being randomized. On the day of cortisol collection, participants were asked to collect one saliva sample 45 minutes after awakening in the morning and one saliva sample at 8:00 pm (see Tables 2-4 for average collection times). They were also asked to abstain from behaviors known to contaminate and interfere with cortisol analyses (e.g., eating, exercising, brushing their teeth) for at least 60 minutes before collecting their saliva samples (Gröschl, Wagner, Rauh, \& Dörr, 2001). Participants were required to complete a cortisol log where they recorded the date and times of cortisol collection, their perceived stress level, as well as any behaviors they believed may have interfered with cortisol collection. After each morning and evening collection, participants were instructed to immediately store their samples in the freezer, complete the study questionnaires and cortisol logs, and leave a 
voicemail message with research staff indicating that the saliva samples had been collected. Voicemail messages were time and date stamped to confirm time of collection.

At six months postpartum, participants collected two more of their own saliva samples (one 45 minutes after awakening and one at 8:00 pm), as well as two samples from their infant (45 minutes after awakening and at 8:00 pm), and completed questionnaires and cortisol logs using the same procedures described above. At 18 months postpartum, only women in the CBSM and UC groups took part in the study, collecting two of their own saliva samples as well as two samples from their child and repeating all the data collection steps listed above.

CBSM Intervention-The cognitive behavioral stress management (CBSM) intervention consisted of a 12-week prenatal course that focused on helping women create a healthy physical, social, and psychological environment for themselves and their infants (see Muñoz et al., 2007a for more details on the development of this intervention). The content of the course was taught from a detailed training manual (Muñoz et al., 2007b) and was based on Albert Bandura's social learning theory (1977) and Peter Lewinsohn's behavioral approach to mood management (1992). The key concepts from these theories (i.e., reciprocal determinism and reinforcement) were integrated in order to teach women how to use cognitive behavioral strategies to manage their mood and health, such as recognizing and modifying maladaptive thoughts, increasing positively reinforcing activities (e.g., walking), identifying and increasing positive social networks, and learning parenting strategies to promote child development and secure attachment. These cognitive behavioral strategies have been found to be effective in reducing stress and treating postpartum depression (Butler, Chapman, Forman, \& Beck, 2006; Chabrol et al., 2002). The course also included teaching stress management strategies to help manage the challenges of pregnancy, labor, childbirth, and caring for a newborn (Ramos, Diaz, Muñoz, \& Urizar, 2002). More specifically, women were taught how to become more aware of the physical symptoms of stress and its effect on one's health, as well as the health of their baby. Further, women were taught several stress management techniques, including diaphragmatic breathing, guided imagery, mindfulness-based training, and progressive muscle relaxation. These stress management strategies have been shown to be effective in reducing stress during pregnancy and improving infant birth outcomes (Beddoe \& Lee, 2008).

The 12-week prenatal course was taught by two group facilitators in Spanish and English to six groups of pregnant women (three to eight women in each group). Women were enrolled in the class following completion of their baseline assessments (6-28 weeks of gestation). The course was conducted at the hospital where women were receiving prenatal services. Facilitators consisted of faculty, postdoctoral fellows, and advanced doctoral graduate students in clinical psychology. A licensed clinical psychologist provided weekly supervision to group facilitators through the use of face-to-face supervisory meetings and by reviewing videotapes of the classes to ensure that facilitators covered the key concepts that were outlined for each class. Before the start of the next class, women who missed the previous class were given a one-on-one review session of the material they missed either in person or over the phone by one of the course facilitators. In addition to attending the 12week prenatal course, women in the CBSM group also participated in four booster sessions conducted at approximately one, three, six, and twelve months postpartum. The purpose of these postpartum booster sessions was to review the core materials taught during the 12week prenatal course and to apply these key concepts towards any new challenges that women may have experienced in caring for their newborn child.

Usual Care and Low Risk Comparison Groups-Women in the usual care (UC) and low risk comparison (LRC) groups received regular medical care from their health care 
provider and were provided with information on locally available social services upon request, or if they developed clinical depression, throughout their participation in the study; however, they did not receive CBSM materials at any point during or after the study. Women in the UC group were identified to be at high risk for depression during pregnancy, while women in the LRC group were identified to be at low risk for depression during pregnancy (see depression risk status criteria below).

\section{Measures}

Participants completed questionnaires that assessed for sociodemographic factors (e.g., age, marital status, income, education), perceived stress levels, and mood (i.e., past history of MDEs, current depressive symptoms, and positive and negative affect). All materials were provided in either Spanish or English, based on the participant's preference. Participants also collected salivary cortisol samples during pregnancy and the postpartum period.

Sociodemographic questionnaire-The sociodemographic questionnaire assessed for maternal characteristics, such as age (in years), number of weeks pregnant, parity status, total years of education, annual household income ( $\$ 0-\$ 20 \mathrm{~K}, \$ 21-\$ 30 \mathrm{~K}, \$ 31 \mathrm{~K}$ and higher), marital status (married/living with a partner, single), primary language spoken at home (Spanish, English), country of birth, and age when they immigrated to the U.S. (in years). The group facilitators that led each class documented the number of classes attended by each participant in the CBSM group. Child characteristics were also assessed and included the child's gender (girl, boy), number of birth complications, the child's age (in months), and the child's primary language (Spanish, Bilingual, English). Number of birth complications experienced was calculated using a Medical Complication Index, which consisted of adding nine birth complication factors together, including the presence of very low or very high birth weight ( $<2499$ grams or $>4000$ grams), gestational age risk ( $<37$ weeks or $>42$ weeks), low APGAR scores at one minute $(<6)$, preeclampsia/hypertension, breech birth, csection delivery, anemia, oxygen resuscitation required, and having an inpatient stay greater than 3 days. This information was collected from a review of the infant's medical record. Each birth complication was given a score of 0 or 1 , depending on the absence or presence of that risk factor, for a total possible score ranging from 0 to 9 . Higher scores represent the presence of more medical complications at birth. Whether or not infants were breastfed (yes/ no) was also assessed from a review of the infant's medical record.

Salivary Cortisol-Mothers collected their saliva using a Salivette sampling device (Salimetrics, LLC) to obtain levels of cortisol during pregnancy, and at six and 18 months postpartum. The salivette device consisted of a small, absorbent cotton swab, which mothers chewed for at least two minutes, or until the cotton swab became soaked with saliva, and then placed the cotton swab into a small plastic tube. To collect their infant's saliva at six and 18 months postpartum, mothers were instructed to use a plastic pipette device that was used to suction saliva from inside the infant's cheek into a microvial storage container (Salimetrics, LLC). Both the salivette and microvial container were then stored at $-20^{\circ} \mathrm{C}$ in the participant's freezer until a member of the research staff collected them. Once these samples were returned to the hospital, they were analyzed in the hospital's laboratory with all procedures being kept constant across samples. After being thawed for biochemical analysis, the samples were centrifuged and salivary cortisol was analyzed using a timeresolved immunoassay with fluorescence detection as described in detail elsewhere (Dressendörfer et al., 1992). All of the samples were assayed in duplicate and averaged. Intra- and interassay variability were both under $8 \%$, with a minimum detectable level of $0.02 \mu \mathrm{g} / \mathrm{dL}$. The cortisol values reported during pregnancy and at six and 18 months postpartum included morning and evening cortisol levels, average cortisol levels, and diurnal cortisol slope (see analyses section below for how these values were computed). 
Perceived Stress-Perceived stress was assessed by a one-item stress question where participants were asked to rate their stress level on a 100-point visual analog scale $(0=\mathrm{Not}$ at all stressed, $100=$ Extremely stressed) to represent how stressed they felt at the time of each cortisol collection (Cline, Herman, Shaw, \& Morton, 1992). The higher the score, the more stress the woman reported experiencing. At 18 months postpartum, mothers were also asked to rate their child's stress level on a 100-point visual analog scale $(0=N o t$ at all stressed to $100=$ Extremely stressed) to represent how stressed they felt their child was at the time of each cortisol collection. A visual analog scale was used to assess perceived stress because it could be administered simply and easily without being too burdensome for women during pregnancy and the postpartum period. Adequate reliability and validity of the visual analog scale has been demonstrated in previous studies with medical patients (Cline et al., 1992; Gift, 1989). Both morning and evening stress ratings, as well as the average of these two ratings, were used and reported in the current study.

Maternal Mood-Depression risk status was determined through the use of two screening questionnaires: 1) the Maternal Mood Screener (MMS; Le \& Muñoz, 1998); and 2) the Center for Epidemiologic Studies - Depression Scale (CES-D; Radloff, 1977). The MMS is an 18-item measure, modeled after the items of the Diagnostic Interview Schedule (Robins, Helzer, Croughan, \& Ratcliff, 1981). The MMS assesses whether the respondent has experienced five of the nine Major Depressive Episode (MDE) symptoms listed in Criterion A of the DSM-IV for a period of at least two weeks and whether the symptoms have interfered significantly with their life or activities (Criterion C of the DSM-IV; American Psychiatric Association, 1994). The MMS was designed to account for somatic symptoms that are common during pregnancy and was used in the current study to screen for either a current or prior history of MDE. If women met these MDE criteria in the past two weeks, they were identified as being currently depressed and referred for treatment. If women had met these MDE criteria sometime in their lifetime, not including the past two weeks (i.e., yes/no; dichotomous variable), they were considered to be at high risk for depression. In contrast, if women had never met these MDE criteria in their lifetime, they were considered to be at low risk for depression. The MMS has been found to agree well with the PRIMEMD (Spitzer et al., 1994), yielding kappas of 0.75 in intervieweradministered versions of this measure (Muñoz et al., 1999). It has also been found to have good predictive validity, with pregnant women with a past MDE history demonstrating significantly higher MDE incidence rates than women without a past MDE history (18-27\% vs. 0-3\%, respectively) during the postpartum period (Le, Muñoz, Soto, Delucchi, \& Ghosh Ippen, 2004).

The CES-D is a 20-item, self-report measure assessing for affective and somatic symptoms of depression during the past week. Each item is rated on a 4-point scale, with higher scores (range $=0$ to 60) reflecting greater symptoms of depression. A cut-off score of 16 is traditionally used for the initial screening of significant depressive symptoms and was used in the current study to identify women who were considered to be at high risk for depression (see Le et al., 2004). The CES-D was administered during pregnancy, and at six and 18 months postpartum, demonstrating good to excellent internal consistencies across the three assessment periods ( $a=0.85$ to 0.92 ) and are consistent with previous estimates for the general U.S. and Latino populations (Guarnaccia et al., 1989; Zich et al., 1990).

The Positive and Negative Affect Schedule (PANAS; Watson, Clark \& Tellegen, 1988) is a 20-item self-report measure assessing one's current positive (e.g., attentive, enthusiastic) and negative (e.g., nervous, distressed) mood state (10 items each). Each item is rated on a 5-point scale $(1=$ Very slightly or not at all to $5=$ Extremely $)$, with higher scores (average score calculation $=$ total score $/ 10$ items; range $=1$ to 5 ) reflecting a greater frequency of positive or negative mood states, respectively. In prior studies, the PANAS has demonstrated good reliability, as well as good discriminate validity with general depression 
and anxiety measures (Crawford \& Henry, 2004). Cronbach's alpha for the the current study was 0.81 during pregnancy, 0.81 at six months postpartum, and 0.88 at 18 months postpartum.

\section{Statistical Analyses}

The distribution of morning and evening cortisol levels for the CBSM, UC, and LRC groups was positively skewed, and so a logarithmic transformation (base 10, cortisol values converted from $\mu \mathrm{g} / \mathrm{dL}$ to $\mathrm{nmol} / \mathrm{L}$ ) was conducted to ensure that scores approximated a normal distribution. These cortisol log scores were subsequently used in all analyses. Both absolute (morning and evening cortisol values) and daily average cortisol (average of morning and evening cortisol) were computed and used in all analyses. In addition, the difference between morning and evening cortisol values was computed to calculate diurnal changes in cortisol slope (morning - evening cortisol values), with larger numbers representing a more normal diurnal slope (i.e., the presence of higher cortisol levels in the morning and a decrease in cortisol levels in the evening) and smaller numbers representing a flatter diurnal cortisol slope. Flatter diurnal cortisol slopes have been associated with several health problems during the postpartum period, including increased anxiety in mothers and low birthweight and impaired sleep among infants (Gunnar \& Vazquez, 2001; Kivlighan, DiPietro, Costigan, and Laudenslager, 2008). Stress ratings were also averaged given that morning and evening stress levels were positively correlated with one another $(r=0.54, p$ $<.001)$.

Pearson's chi-squared and ANOVAs were conducted for categorical and continuous variables, respectively, to assess for between group differences (i.e., CBSM, UC, and LRC groups) on study characteristics (Table 1). Pearson product-moment correlations were also performed to help identify associations among sociodemographic factors (e.g., marital status, number of children, infant gender) and our stress and mood variables (cortisol, perceived stress, depression, and positive and negative affect).

Paired comparison t-tests were conducted to assess for within group changes in stress (i.e., cortisol, perceived stress) and mood (i.e., depression, positive and negative affect) levels from pregnancy to six months postpartum, as well as from six to 18 months postpartum. Furthermore, analysis of covariance (ANCOVA) was used to assess for between group differences (i.e., CBSM, UC, and LRC groups) on stress (i.e., cortisol, perceived stress) and mood (i.e., depression, positive and negative affect) levels during pregnancy and at six months postpartum (general linear model; Spector, Goodnight, Sall, \& Sarle, 1985), whereas independent samples $t$ test analyses were used to assess for group differences (i.e., CBSM vs. UC groups) on stress and mood levels at 18 months postpartum. The main effects for group condition were evaluated, with certain sociodemographic variables serving as covariates (i.e., past history of depression and annual combined income for maternal cortisol; and maternal cortisol and maternal age for infant cortisol, respectively) based on significant associations found between these covariates and maternal and infant cortisol levels in the Pearson product-moment correlation analyses.

Exploratory ANCOVA analyses were performed to test for significant two way interactions [i.e., group condition by sociodemographic characteristics (e.g., maternal age, past history of depression)] that could influence intervention effects on maternal and infant cortisol, respectively. Interaction terms of $p<.05$ were considered to be significant. A median split of continuous variables (e.g., maternal age) was then used to illustrate significant moderation effects by sociodemographic characteristics. The least-squares means method was used to compare group means for all significant effects. Casewise deletion of missing data was used in all analyses. 


\section{Results}

\section{Participants}

Of the 86 women who participated in cortisol collection for the Mothers and Babies: Mood and Health Project, 57 women (66\%) were identified as being at high risk for depression during pregnancy and randomized to the CBSM intervention $(n=24)$ or the UC group $(n=$ 33). In addition, 29 women were identified as being at low risk for depression during pregnancy and took part in the LRC group. A description of the study sample is shown in Table 1.

Overall, women in all three groups were similar on most sociodemographic characteristics. Participants were approximately 25 years of age (range $=18-35$ years), in their $16^{\text {th }}$ week of pregnancy (range $=6-27$ weeks), and had less than 12 years of education $(M=10$ years, $S D$ =3.4). Of these, the majority were Spanish-speaking Latina women $(80 \%)$ who were born in Mexico/Central America (78\%), and were on average 19 years of age when they immigrated to the U.S. $(S D=7.0)$. Approximately $20 \%$ of women spoke primarily English and were of various ethnic backgrounds (i.e., 6\% African-American, 2\% Caucasian, $11 \%$ Other). Most women were married or living with a partner (77\%) and had an annual household income under $\$ 30,000$ (87\%). Chi square analyses demonstrated that women in the LRC group were more likely to have an annual family income less than $\$ 20,000$ per year than women in the CBSM and UC groups $\left(\chi^{2}=14.5, p<.01\right)$. No other significant differences were observed between the CBSM, UC, and LRC groups on other maternal characteristics at baseline, including gestational age $(F=0.31, p=0.74)$.

Most women had previously experienced childbirth (60\%; range $=0-6$ children). In addition, infants born during the study were mostly boys (55\%), Spanish-speaking (67\%), breastfed (94\%), and, on average, had experienced at least one medical complication at birth (range = $0-5$ birth complications). The most common medical complications experienced by infants included needing oxygen at birth (43\%), having an inpatient stay of three or more days (18\%), being delivered by C-section (17\%), having a very low or very high birthweight (17\%), having a low Apgar score at one minute (15\%), and having anemia (14\%).

\section{Cortisol, Stress, and Mood Levels During Pregnancy}

Adherence to Study Protocol-During pregnancy, 84 of the 86 participants (98\%) adhered to cortisol collection procedures by recording the dates and times when saliva samples were collected at home and leaving a voicemail message to research staff when the protocol was completed. Similar adherence rates were observed at six (96\%) and $18(85 \%)$ months postpartum (see Tables 2-4 for average collection times; range of missing data: 09\%). Based on information from their cortisol logs, none of the participants reported consuming any foods, exercising, or taking any medications known to interfere with cortisol levels prior to cortisol collection at any point during the study.

Prenatal Cortisol and Stress-Pearson correlation analyses revealed that cortisol levels were higher among women with greater gestational age $(r=0.26, p<.05)$. ANCOVA analyses demonstrated that women randomized to the CBSM group had significantly lower morning, evening, and average cortisol levels at baseline compared to women in the LRC group, even after adjusting for gestational age and time of cortisol collection $(p<.05)$. Therefore, baseline cortisol levels were included as a covariate for ANCOVA analyses conducted at six and 18 months postpartum. No other significant group differences were observed for salivary cortisol or self-reported stress levels. 
Prenatal Mood-In general, women with high depressive symptoms during pregnancy also rated their stress levels as being high $(r=0.23, p<.05)$ and were more likely to have a past history of depression $(t=-2.3, p<.05)$. Positive affect was higher among women with more education $(r=0.29, p<.01)$, low depressive symptoms $(r=-0.32, p<.01)$, and no past history of depression $(t=2.3, p<.05)$. In contrast, negative affect was higher among women with more perceived stress $(r=0.39, p<.001)$ and depressive symptoms $(r=0.28, p<.05)$. As would be expected, none of the women in the LRC group had a past history of depression and had lower overall depressive symptoms during pregnancy, relative to women in the CBSM and UC groups $(F=21.1, p<.001)$. Women in the LRC group also reported significantly higher positive affect during pregnancy compared to women in the UC group $(F=4.1, p<.05)$; no significant differences were observed with the CBSM group.

\section{Cortisol, Stress, and Mood Levels at Six Months Postpartum}

Study Retention-At six months postpartum, 75 of the 86 participants (87\%) completed the study protocol. Seven participants from the LRC group and four participants from the UC group had incomplete data. Of these 11 participants, two had lost their baby during pregnancy, three moved away, two were not able to be contacted, and four did not return their cortisol materials in time. These 11 participants did not differ from those that completed the study protocol by age, number of children, education, income, or prenatal depression level. On average, women in the CBSM group attended 7 out of 12 classes during pregnancy and 2 out of 4 booster sessions over the first postpartum year (see Table 1). No sociodemographic characteristics were associated with class attendance during pregnancy, however, women who had more children attended fewer postpartum booster sessions $(r=-0.54, p<.01)$.

Maternal Cortisol and Stress Levels-At six months postpartum, higher average cortisol levels were observed among women with "flatter" or more abnormal diurnal cortisol patterns (i.e., smaller decrease in cortisol levels across the day; $r=-0.31, p<.01$ ) and among women who experienced giving birth to infants with birth complications $(r=0.23, p$ $=.05$ ). As expected, paired sampled t-test analyses revealed that women's average cortisol levels significantly dropped from pregnancy to six months postpartum $(t=3.9, p<.001)$, with steeper decreases in cortisol observed among women in the $\operatorname{LRC}(t=4.4, p<.001)$ and UC groups $(t=2.4, p<.05)$. Diurnal cortisol patterns also became more normal (i.e., steeper cortisol decrease from morning to evening) over this time period $(t=-1.8, p=.07)$, with more normal diurnal cortisol patterns observed among women randomized to the CBSM group $(t=-1.8, p=.08)$. No between group differences were observed for maternal cortisol at six months postpartum.

Pearson correlation analyses demonstrated that women who experienced high perceived stress during pregnancy also experienced high stress levels at six months postpartum ( $r=$ $0.32, p<.05)$. In turn, higher perceived stress at six months postpartum was associated with higher cortisol levels $(r=0.31, p<.05)$ and higher depressive symptoms $(r=0.58, p<.001)$ at six months postpartum. Paired sampled t-test analyses showed that only women randomized to the CBSM group reported higher perceived stress from pregnancy to six months postpartum $(t=-3.8, p<.01)$. Further, ANOVA analyses demonstrated that women in the CBSM group had significantly higher perceived stress levels than women in the LRC group at six months postpartum $(p<.05$; Table 3$)$. Contrary to our hypothesis, women in the CBSM group who attended more prenatal classes reported higher perceived stress levels at six months postpartum than women in the CBSM group who attended fewer prenatal classes $(r=0.49, p<.05)$; however, those who attended more prenatal classes also reported having less negative affect $(r=-0.49, p<.05)$. 
Infant Cortisol-Pearson correlation analyses showed that cortisol levels were higher among infants of mothers with high cortisol levels $(r=0.52, p<.001)$ and more abnormal diurnal cortisol patterns at six months postpartum $(r=-0.25, p<.05)$, among infants with more siblings $(r=0.29, p<.05)$, among infants of older mothers compared to infants of younger mothers $(r=0.25, p<.05)$, and among boys compared to girls $(t=-2.0, p<.05)$. Cortisol levels were also higher among infants who required oxygen during birth $(r=0.36, p$ $<.01)$ and among infants who were not breastfed $(r=0.31, p<.05)$. Further, independent samples $t$ test analyses demonstrated that infants of mothers with a past history of depression had higher morning cortisol levels than infants of mothers without a past history of depression $(t=-2.4, p<.05)$. As hypothesized, ANCOVA analyses revealed lower average cortisol levels among infants of women in the CBSM $(M=0.76, S E=0.07)$ and LRC $(M=$ $0.77, S E=0.08)$ groups, relative to infants of women in the UC group $(M=1.0, S E=0.06)$, after adjusting for maternal cortisol at six months postpartum and maternal age $(F=9.6, p$ $<.001$ ). Infants of women in the CBSM group who attended more prenatal classes had lower evening cortisol levels than infants of women in the CBSM group who attended fewer prenatal classes $(r=-0.43, p=.05)$. A significant group by maternal age interaction was also found (median age $=24$ ), with infants of younger mothers (i.e., 18-23 years of age) in the CBSM group having lower cortisol levels at six months postpartum $(M=0.22 \mu \mathrm{g} / \mathrm{dL}, S E=$. $07)$ than infants of younger mothers in the UC group $(M=0.42 \mu \mathrm{g} / \mathrm{dL}, S E=.06 ; F=4.0, p$ $<.05)$; however, this group difference was not observed among infants of older mothers (i.e., 24 years of age or older; see Figure 1).

Maternal Mood-Pearson correlation analyses revealed that women with high depressive symptoms and positive affect during pregnancy also had high depressive symptoms ( $r=$ $0.34, p<.05)$ and positive affect $(r=0.29, p<.05)$ at six months postpartum, respectively. Independent samples $t$ test analyses showed that mothers with a past history of depression had less positive affect at six months postpartum than mothers without a past history of depression $(t=2.6, p<.05)$. In addition, single mothers had more negative affect at six months postpartum compared to mothers who were married or living with a partner $(t=-2.6$, $p<.01)$. For all women in the sample, paired sampled t-test analyses demonstrated that positive affect significantly decreased $(t=6.1, p<.001)$ while negative affect significantly increased $(t=-6.4, p<.001)$ from pregnancy to six months postpartum. ANOVA analyses revealed that women in the CBSM group reported significantly lower positive affect at six months postpartum compared to women in the LRC group $(F=4.0, p<.05)$; no significant group differences were observed for women in the UC group.

\section{Cortisol, Stress, and Mood Levels at Eighteen Months Postpartum}

Study Retention-Given the nature of the study design, only women in the CBSM and UC groups were followed at 18 months postpartum. Of these 53 women, 46 (87\%) completed the study protocol. Seven participants from the UC group had incomplete data. Of these seven participants, two moved away and five were not able to be contacted. These seven participants did not differ from those that completed the study protocol at 18 months postpartum by age, number of children, education, or income level. However, noncompleters tended to have lower levels of depression at six months postpartum than women who completed the study protocol $(t=-2.4, p<.05)$.

Maternal Cortisol and Stress Levels-Independent samples t test analyses showed that women with a past history of depression had higher cortisol levels at 18 months postpartum than women without a past history of depression $(t=-1.9, p=.06)$. Higher cortisol levels were also observed among English-speaking women compared to Spanishspeaking women $(t=-2.7, p<.05)$, as well as among women with a higher annual family income $(r=0.37, p<.05)$. Paired sampled t-test analyses revealed that women's diurnal 
cortisol patterns became more normal from six to 18 months postpartum $(t=-2.2, p<.05)$, particularly among women randomized to the CBSM group. In addition, women in the CBSM group who attended more prenatal classes and postpartum booster sessions had lower average cortisol levels at 18 months postpartum than women who attended fewer classes $(r=$ $-0.36, p<.05)$. ANCOVA analyses revealed lower average cortisol levels among women in the CBSM group $(M=0.59, S E=0.08)$, relative to women in the UC group $(M=0.82, S E=$ $0.08)$, after adjusting for past history of depression and annual family income $(F=5.1, p<$. 01). A significant group by past history of depression interaction was also found, such that women in the CBSM group with a past history of depression $(M=0.23 \mu \mathrm{g} / \mathrm{dL}, S E=.03)$ had lower average cortisol levels at 18 months postpartum than women in the UC group with a past history of depression $(M=1.08 \mu \mathrm{g} / \mathrm{dL}, S E=.41 ; F=4.3, p<.05)$; however, this group difference was not observed among women without a past history of depression (see Figure 2).

Women with high perceived stress levels during pregnancy and at six months postpartum also reported high stress levels at 18 months postpartum $(r=0.45, p<.05 ; r=0.42, p<.05$, respectively). Higher stress levels were also reported by women who experienced giving birth to infants with more birth complications $(r=0.33, p<.05)$, among women with a higher annual family income $(r=0.34, p<.05)$, and among women with fewer children $(r=$ $-0.30, p=.06)$. Paired sampled t-test analyses showed that only women randomized to the CBSM group reported lower morning stress levels from six to 18 months postpartum ( $t=$ $2.5, p<.05)$. However, women in the CBSM group who attended more prenatal classes reported higher perceived stress at 18 months postpartum compared to women in the CBSM group who attended fewer prenatal classes $(r=0.52, p<.05)$. No significant between group differences were observed for perceived stress at 18 months postpartum.

Child Cortisol and Stress Levels-Pearson correlation analyses showed that children who had high average cortisol levels and more abnormal diurnal cortisol patterns at six months postpartum had high cortisol levels at 18 months postpartum $(r=0.35, p=.05 ; r=$ $-0.34, p=.06$, respectively). Furthermore, children who had high cortisol levels at 18 months postpartum also tended to have more abnormal (i.e., "flatter") diurnal cortisol patterns at 18 months postpartum $(r=-0.39, p<.05)$. Abnormal diurnal cortisol patterns were observed among children of mothers with more depressive symptoms $(r=-0.34, p<$. $05)$, children of older mothers $(r=-0.31, p=.07)$, and children who experienced less medical complications at birth $(r=0.45, p<.01)$. Children of women in the CBSM group who attended more prenatal classes had lower average cortisol levels at 18 months postpartum than children of women in the CBSM group who attended fewer prenatal classes $(r=-0.54, p<.05)$. No significant between group differences were observed for child cortisol at 18 months postpartum.

Mothers with more negative affect during pregnancy rated their child as being more stressed at 18 months postpartum $(r=0.50, p<.01)$. In addition, mothers who reported feeling more stressed at six and 18 months postpartum tended to rate their child as being more stressed at 18 months postpartum $(r=0.37, p<.05 ; r=0.70, p<.001$, respectively). High maternal ratings of child stress levels were also observed among children who experienced more medical complications at birth $(r=0.34, p<.05)$, children who had fewer siblings $(r=-0.33$, $p<.05)$, children of younger mothers $(r=-0.32, p<.05)$, and girls compared to boys $(t=$ $2.4, p<.05)$. No significant between group differences were observed for child stress levels at 18 months postpartum.

Maternal Mood-Paired sampled t-test analyses demonstrated that women's positive affect significantly increased $(t=-5.7, p<.001)$ and their negative affect significantly decreased $(t=6.6, p<.001)$ from six to 18 months postpartum. Pearson correlation analyses 
showed that women with high depressive symptoms during pregnancy also had high depressive symptoms at 18 months postpartum $(r=0.48, p<.01)$. In contrast, women with high positive affect during pregnancy reported lower depressive symptoms at 18 months postpartum $(r=-0.37, p<.05)$. Finally, women with high negative affect at six months postpartum also reported high negative affect at 18 months postpartum $(r=0.43, p<.05)$. No significant between group differences were observed for maternal mood at 18 months postpartum.

\section{Discussion}

\section{CBSM Intervention Effects on Maternal Cortisol}

Despite the adverse effects of stress on maternal and infant health, few studies have examined the efficacy of stress management interventions on regulating cortisol and stress levels among mothers and their infants. Results from the current pilot study are one of the first, to our knowledge, to prospectively examine whether a prenatal cognitive behavioral stress management (CBSM) intervention would be effective in regulating salivary cortisol and self-reported stress levels among low-income mothers and their infants at six and 18 months postpartum. Contrary to our hypothesis, no significant group differences were found for maternal cortisol levels at six months postpartum among women receiving the CBSM intervention, relative to women in a usual care (UC) and low risk comparison (LRC) group. Women in our study reported a general increase in perceived stress and negative affect (as well as a decrease in positive affect) from pregnancy to six months postpartum. These results are consistent with previous studies which have documented that although there is often much pleasure and happiness in the birth of a new baby, several maternal stressors (e.g., fatigue, lack of social support, marital conflict) may also arise during the early postpartum period, causing a stressful transition in women's social roles and parenting responsibilities and, therefore, potentially nullifying any CBSM intervention effects on maternal cortisol levels during this time period (Groer, Davis, \& Hemphill, 2002).

Our results also showed that women in the CBSM group reported feeling more stressed at six months postpartum. These results may be due to the amount of information they received as part of the CBSM intervention during pregnancy, which was intended to better prepare them for the care of their newborn baby, but may have also raised their awareness about how critical the early postpartum period is to the development of their baby. However, our results also indicated that women in the CBSM group had less negative affect at six months postpartum, suggesting that although the CBSM intervention may have raised their anxieties about motherhood, it was also effective in helping to regulate their negative mood states, which have been associated with increasing an infant's risk for developing depressive symptoms, behavioral problems, and developmental deficits later in childhood (Misri \& Kendrick, 2007).

Of note, although no significant group differences were observed for average maternal cortisol levels at six months postpartum, women in the CBSM group demonstrated more normal diurnal cortisol patterns (i.e., the presence of higher cortisol levels in the morning and a decrease in cortisol levels in the evening) from pregnancy $(M=59 \%$ decrease in diurnal cortisol) to six months postpartum ( $M=78 \%$ decrease in diurnal cortisol). More normal diurnal cortisol patterns during this time period were a result of a significant decrease in evening cortisol in the CBSM group from pregnancy to six months postpartum. This trend continued from six to 18 months postpartum, with women in the CBSM group demonstrating continued normalization of their diurnal cortisol patterns (i.e., $63 \%$ vs. $80 \%$ decrease in diurnal cortisol, respectively) due to a significant decrease in evening cortisol. These results suggest that the CBSM intervention was effective in decreasing women's risk for developing abnormal (i.e., flatter) diurnal cortisol patterns during the early postpartum 
period, which have been associated with several health problems for women, including increased risk for cardiovascular disease and anxiety (Kivlighan et al., 2008; Rosmond \& Björntorp, 2000). In the current study, our results showed that women who had abnormal diurnal cortisol patterns had infants with higher cortisol levels at six months postpartum, providing evidence for how physiological stress patterns in mothers may affect the stress levels of their baby.

As hypothesized, women in the CBSM group had lower cortisol levels at 18 months postpartum relative to mothers in a usual care (UC) group. To our knowledge, this is one of the first studies to demonstrate long-term prenatal CBSM effects on maternal cortisol later in the postpartum period, as previous studies have reported intervention effects immediately post-birth up to six months postpartum (Beddoe \& Lee, 2008; Field, 1998; Field et al., 2000; Field et al., 2004; Wesley, 2006). These long-term intervention effects may be due to the postpartum booster sessions that were provided for women in the CBSM group (up to 12 months postpartum), which focused on reviewing and applying key CBSM concepts to stressors that women were experiencing during the postpartum period. Women also reported feeling less stressed (particularly women in the CBSM group), as well as less negative affect and more positive affect from six to 18 months postpartum, suggesting that women may have had time to adjust to their parenting role and engage in CBSM strategies to better manage their stress during this time period.

Previous studies have shown individuals with a past history of depression, as well as infants of mothers with a past history of depression, to have higher cortisol levels (Brennan et al., 2008; Young, Aggen, Prescott, \& Kendler, 2000). These findings were confirmed in the current study, with women with a past history of depression having higher cortisol levels at 18 months postpartum, and their infants having higher cortisol levels at six months postpartum, relative to women without a past history of depression. However, it is unclear from these results whether higher cortisol levels among women with a past history of depression were due to continued HPA axis activation that began prior to pregnancy or if having a past history of depression made these women more vulnerable to increased HPA axis activation during the postpartum period. Furthermore, elevations in infant cortisol among mothers with a past history of depression may provide some support for the biological transmission of altered HPA modulation from mother to child during pregnancy, as maternal cortisol levels have been shown to readily cross the placenta during fetal development and affect infant cortisol (Brennan et al., 2008). Interestingly, results from the current study demonstrated that the CBSM intervention was particularly effective in reducing cortisol for women with a past history of depression and suggest that future studies should target this subgroup of mothers and infants for prenatal CBSM interventions in order to prevent future adverse health outcomes (e.g., Essex et al., 2002).

\section{CBSM Intervention Effects on Infant Cortisol}

As expected, infants of mothers in the CBSM and LRC groups had lower cortisol levels at six months postpartum compared to infants of mothers in the UC group. This is one of the few studies to demonstrate prenatal CBSM intervention effects on infant cortisol levels during the postpartum period. These results are consistent with a previous study that examined the effects of a 3-month postpartum lifestyle intervention (i.e., depressed mothers receiving social services, learning relaxation techniques, and receiving massage therapy and mother-infant interaction coaching) on infant cortisol. Following the intervention, infants of depressed mothers who received the intervention had cortisol levels that were similar to those of infants of non-depressed mothers (control group) at six months postpartum (Field et al., 2000). Collectively, these results suggest that teaching mothers CBSM strategies during pregnancy or the early postpartum period are effective in helping to regulate infant cortisol levels.

Psychoneuroendocrinology. Author manuscript; available in PMC 2012 November 01. 
Athough mothers in the CBSM group were more stressed at six months postpartum, this did not seem to affect their infant's cortisol levels, suggesting that mothers in the CBSM group may have directed their parenting-related stress towards taking care of their infant's needs before their own. Of note, maternal age was found to be a significant moderator of CBSM group effects on infant cortisol, with infants of younger mothers (i.e., 18-23 years of age) in the CBSM group demonstrating lower cortisol levels at six months postpartum than infants of younger mothers in the UC group (no group differences were observed among infants of older women in the study -24 years of age or older). These results suggest that younger women, in particular, may greatly benefit from learning CBSM strategies that are focused on managing the challenges of motherhood as well as meeting the needs of their baby during the early postpartum period. However, further studies are needed to examine the moderating effects of maternal age on maternal and infant cortisol levels given the different resources (e.g., financial, social support) and competing demands (e.g., caring for other children) that younger and older mothers may face (Tu, Lupien, \& Walker, 2006).

\section{Study Limitations}

The findings presented should be interpreted with some degree of caution given several study limitations. First, given that the majority of our sample consisted of low-income Latina women (80\% Spanish-speaking), the results may not be generalizeable to women and children from other socioeconomic or ethnic backgrounds. However, this is one of the first studies to examine the effects of a CBSM intervention on the stress levels of this growing and important population. Second, our sample size was relatively small $(n=86)$ as a result of cases in which cortisol data for mothers and their children were incomplete and, therefore, were eliminated from further analyses. More specifically, a total of seven participants from the LRC group and nine participants from the UC group were lost to follow-up throughout the duration of the study ( $\sim 87 \%$ retention rate). Future studies with pregnant women may want to incorporate attention-control groups (e.g., prenatal health education) to minimize differential drop-out rates across these randomization arms. Additional studies in this research area, with larger sample sizes, would aid in supporting our cortisol findings, as well as further our understanding of the impact of a prenatal CBSM intervention on levels of perceived stress and mood among mothers and their children. Although women in each of our groups did not significantly differ by gestational age at the time that baseline cortisol was collected, future studies may want to stratify their randomization groups by gestational age since variability in this factor at baseline can affect prenatal cortisol outcomes. Finally, given that our protocol adherence measures for salivary cortisol collection were largely based on self-report, it is difficult to accurately determine how adherent mothers were with the cortisol collection instructions. However, time- and date-stamped voicemail messages from participants upon completion of each cortisol collection period helped to confirm that the majority of women were adherent in collecting their salivary cortisol at the designated times (85\%-98\% adherence rates; 0-9\% missing data).

\section{Implications}

In summary, our results suggest that teaching low-income women CBSM strategies during pregnancy is effective in regulating maternal and infant cortisol levels up to 18 months postpartum, particularly among women with a past history of depression or among younger mothers. These results potentially have long-term implications for the health of mothers and their infants in helping to prevent the onset of stress-related health complications that have been associated with elevated cortisol levels during pregnancy. Despite the disproportionate number of premature births and low birthweight babies observed among low-income mothers, less than $25 \%$ of these women report actively engaging in stress management during pregnancy due to lack of awareness of how important managing one's stress levels 
can be for the health of the mother and her baby (Gharaibeh et al., 2005; Hawkins et al., 1998). Given these disparities in birth outcomes and access to preventive interventions, research studies are needed to reach underserved women during pregnancy and provide them with the necessary skills and knowledge to facilitate stress management. Prenatal CBSM interventions can be designed to help women better prepare for the many challenges related to pregnancy, childbirth, and motherhood, and provide a viable alternative to pharmacological approaches to managing maternal stress levels. Furthermore, these interventions can be easily adapted in community-based health settings to reach traditionally underserved populations that may be at increased risk for adverse pre- and postnatal health outcomes. However, carefully controlled, longitudinal studies are needed to demonstrate the potential long-term health benefits that these interventions may have for mothers and their children (e.g., child's cognitive functioning), as well as examine whether these health benefits are a result of cortisol regulation during pregnancy and the early postpartum period. Furthermore, further research is needed to identify the critical threshold by which elevated cortisol levels lead to adverse health outcomes for mothers and their children.

\section{Acknowledgments}

This study was conducted in part in the GCRC at San Francisco General Hospital and supported by Grant 5-MO1RR00083, Division of Research Resources, National Institutes of Health. The authors would like to acknowledge Bobbye Chang for conducting the cortisol assays. The preparation of this paper was supported by a grant from the Division of Research Resources at the National Institute of Health (5-MO1-RR00083) and a grant by the University of California Office of the President's Committee on Latino Research for the UCSF/San Francisco General Hospital Latino Mental Health Research Program (Ricardo F. Muñoz, PI). Additional support was provided by grants from the National Institute of Mental Health (MH 596056, Ricardo F. Muñoz, PI), the Research and Evaluation Allocation Committee of the UCSF School of Medicine, the University of California Mexico-U.S. (UC MEXUS) research program, and by private donations from Dr. Cloyce L. Duncan and Dr. Gwendolyn Evans for the Mamás y Bebés/Mothers and Babies: Mood and Health Project. At the time this study was conducted, G. Urizar was funded as a postdoctoral fellow by the UCSF Clinical Psychology Training Program.

\section{References}

American Psychiatric Association. Diagnostic and statistical manual of mental disorders. 4. Washington, DC: American Psychiatric Association; 1994.

Antoni MH. Stress management effects on psychological, endocrinological, and immune functioning in men with HIV infection: Empirical support for a psychoneuroimmunological model. Stress. 2003; 6(3):173-188. [PubMed: 13129811]

Ashman SB, Dawson G, Panagiotides H, Yamada E, Wilkinson CW. Stress hormone levels of children of depressed mothers. Development and Psychopathology. 2002; 14:333-349. [PubMed: 12030695]

Austin MP, Leader L. Maternal stress and obstetric and infant outcomes: Epidemiological findings and neuroendocrine mechanisms. The Australian \& New Zealand Journal of Obstetrics \& Gynaecology. 2000; 40(3):331-337.

Bandura, A. Social learning theory. Englewood Cliffs, NJ: Prentice-Hall; 1977.

Barlow, DH.; Cerny, JA. Psychological Treatment of Panic. New York, NY: Guilford Press; 1988.

Beck AT. Cognitive therapy: A 30-year retrospective. American Psychologist. 1991; 46(4):368-375. [PubMed: 2048795]

Beddoe AE, Lee KA. Mind-body interventions during pregnancy. Journal of Obstetric, Gynecologic, and Neonatal Nursing. 2008; 37:165-175.

Brennan PA, Pargas R, Walker EF, Green P, Newport DJ, Stowe Z. Maternal depression and infant cortisol: Influences of timing, comorbidity and treatment. Journal of Child Psychology and Psychiatry. 2008; 49(10):1099-1107. [PubMed: 18492036]

Butler AC, Chapman JE, Forman EM, Beck AT. The empirical status of cognitive-behavior therapy: A review of meta-analyses. Psychology Review. 2006; 26(1):17-31. 
Chabrol H, Teissedre F, Saint-Jean M, Teisseyre N, Roge B, Mullet E. Prevention and treatment of post-partum depression: A controlled randomized study on women at risk. Psychological Medicine. 2002; 32:1039-1047. [PubMed: 12214785]

Cline ME, Herman J, Shaw E, Morton RD. Standardization of the visual analogue scale. Nursing Research. 1992; 41:378-379. [PubMed: 1437591]

Crawford JR, Henry JD. The Positive and Negative Affect Schedule (PANAS): Construct validity, measurement properties and normative data in a large non-clinical sample. British Journal of Clinical Psychology. 2004; 43:245-265. [PubMed: 15333231]

Cruess DG, Antoni MH, McGregor BA, Kilbourn KM, Boyers AE, Alferi SM, Carver CS, Kumar M. Cognitive-behavioral stress management reduces serum cortisol by enhancing benefit finding among women being treated for early stage breast cancer. Psychosomatic Medicine. 2000; 62:304308. [PubMed: 10845343]

Da Costa D, Larouche J, Dritsa M, Brender W. Psychosocial correlates of prepartum and postpartum depressed mood. Journal of Affective Disorders. 2000; 59:31-40. [PubMed: 10814768]

Diego MA, Field T, Hernandez-Reif M, Cullen C, Schanberg S, Kuhn C. Prepartum, postpartum, and chronic depression effects on newborns. Psychiatry. 2004; 67(1):63-80. [PubMed: 15139586]

Dressendörfer RA, Kirschbaum C, Rohde W, Stahl F, Strasburger CJ. Synthesis of a cortisol-biotin conjugate and evaluation as a tracer in an immunoassay for salivary cortisol measurement. Journal of Steroid Biochemistry and Molecular Biology. 1992; 43(7):683-692. [PubMed: 1472460]

Essex MJ, Klein MH, Cho E, Kalin NH. Maternal stress beginning in infancy may sensitize children to later stress exposure: Effects on cortisol and behavior. Biological Psychiatry. 2002; 52:776-784. [PubMed: 12372649]

Field T. Early interventions for infants of depressed mothers. Pediatrics. 1998; 102(5):1305-1310. [PubMed: 9794974]

Field T, Diego MA, Dieter J, Hernandez-Reif M, Schanberg S, Kuhn C, Yando R, Bendell D. Prenatal depression effects on the fetus and the newborn. Infant Behavior \& Development. 2004; 27:216229.

Field T, Pickens J, Prodromidis M, Malphurs J, Fox N, Bendell D, Yando R, Schanberg S, Kuhn C. Targeting adolescent mothers with depressive symptoms for early intervention. Adolescence. 2000; 35(138):381-414. [PubMed: 11019779]

Gaab J, Blättler N, Menzi T, Pabst B, Stoyer S, Ehlert U. Randomized controlled evaluation of the effects of cognitive-behavioral stress management on cortisol responses to acute stress in healthy subjects. Psychoneuroendocrinology. 2003; 28:767-779. [PubMed: 12812863]

Gharaibeh M, Al-Ma'aitah R, Al Jada N. Lifestyle practices of Jordanian pregnant women. International Nursing Review. 2005; 52:92-100. [PubMed: 15842321]

Gift AG. Visual analogue scales: Measurement of subjective phenomena. Nursing Research. 1989; 38:286-288. [PubMed: 2678015]

Gitau R, Camerson A, Fisk N, Glover V. Fetal exposure to maternal cortisol. The Lancet. 1998; 352:707.

Goodman SH, Gotlib IH. Risk for psychopathology in the children of depressed mothers: A developmental model for understanding mechanisms of transmission. Psychological Review. 1999; 106(3):458-490. [PubMed: 10467895]

Hawkins JW, Aber CS, Cannan A, Coppinger CM, Rafferty KO. Women's reported self-care behaviors during pregnancy. Health Care for Women International. 1998; 19:529-538. [PubMed: 9849198]

Groer M, Davis M, Hemphill J. Postpartum stress: Current concepts and the possible protective role of breastfeeding. Journal of Obstetrical, Gynecological, and Neonatal Nursing. 2002; 31:411-417.

Gröschl M, Wagner R, Rauh M, Dörr HG. Stability of salivary steroids: The influences of storage, food, and dental care. Steroids. 2001; 66:737-741. [PubMed: 11522335]

Guarnaccia PJ, Angel R, Worobey JL. The factor structure of the CES-D in the Hispanic health and nutrition examination survey: The influences of ethnicity, gender, and language. Social Science \& Medicine. 1989; 29(1):85-94. [PubMed: 2740931]

Gunnar MR. Quality of early care and buffering of neuroendocrine stress reactions: Potential effects on the developing human brain. Preventive Medicine. 1998; 27:208-211. [PubMed: 9578997] 
Gunnar MR, Vazquez DM. Low cortisol and a flattening of expected daytime rhythm: Potential indices of risk in human development. Development and Psychopathology. 2001; 13:515-538. [PubMed: 11523846]

Harris B. Hormonal aspects of postnatal depression. International Review of Psychiatry. 1996; 8:2736.

Hendrick V, Altshuler LL, Suri R. Hormonal changes in the postpartum and implications for postpartum depression. Psychosomatics. 1998; 39(2):93-101. [PubMed: 9584534]

Kivlighan KT, DiPietro JA, Costigan KA, Laudenslager ML. Diurnal rhythm of cortisol during late pregnancy: Associations with maternal psychological well-being and fetal growth. Psychoneuroendocrinology. 2008; 33:1225-1235. [PubMed: 18692319]

Knoches AML, Doyle LW. Long-term outcome of infants born preterm. Baillière's Clinical Obstetrics and Gynaecology. 1993; 7(3):633-651.

Kurstjens S, Wolke D. Effects of maternal depression on cognitive development of children over the first seven years of life. Journal of Child Psychology and Psychiatry \& Allied Disciplines. 2001; 42(5):623-636.

Le, HN.; Muñoz, RF. The Maternal Mood Screener. 1998. [Unpublished measure]. Retrieved October 2, 2005, from http://medschool.ucsf.edu/latino/manuals.aspx

Le HN, Muñoz RF, Soto JA, Delucchi KL, Ippen CG. Identifying risk for onset of Major Depressive Episodes in low-income Latinas during pregnancy and postpartum. Hispanic Journal of Behavioral Sciences. 2004; 26:463-482.

Lewinsohn, PM.; Muñoz, RF.; Youngren, MA.; Zeiss, AM. Control your depression. New York: Fireside Books; 1992.

Lobel M, Cannella DL, Graham JE, DeVincent C, Schneider J, Meyer BA. Pregnancy-specific stress, prenatal health behaviors, and birth outcomes. Health Psychology. 2008; 27(5):604-615. [PubMed: 18823187]

Lundy BL, Jones NA, Field T, Nearing G, Davalos M, Pietro PA, Schanberg S, Kuhn C. Prenatal depression effects on neonates. Infant Behavior \& Development. 1999; 22(1):199-129.

McCool WF, Dorn LD, Susman EJ. The relation of cortisol reactivity and anxiety to perinatal outcome in primiparous adolescents. Research in Nursing \& Health. 1994; 17:411-420. [PubMed: 7972919]

Misri S, Kendrick K. Treatment of perinatal mood and anxiety disorders: A review. Canadian Journal of Psychiatry. 2007; 52(8):489-498.

Muñoz RF, Le HN, Ghosh Ippen C, Diaz MA, Urizar G, Soto J, Mendelson T, Delucchi K, Lieberman A. Prevention of postpartum depression in low-income women: Development of the Mamás $y$ Bebés/Mothers and Babies Course. Cognitive \& Behavioral Practice. 2007a; 14:70-83.

Muñoz, RF.; Le, H.; Gosh-Ippen, C.; Diaz, M.; Urizar, G.; Lieberman, A. The Mothers and Babies Course: Instructor's Manual. 2007b. Retrieved April 20, 2009, from http://www.medschool.ucsf.edu/latino/Englishmanuals.aspx

Muñoz RF, McQuaid JR, González GM, Dimas J, Rosales VA. Depression screening in a women's clinic: Using automated Spanish- and English-language voice recognition. Journal of Consulting and Clinical Psychology. 1999; 67(4):502-510. [PubMed: 10450620]

Muñoz, RF.; Ying, Y. The prevention of depression: Research and practice. Baltimore, MD: Johns Hopkins University Press; 1993.

Ponirakis A, Susman EJ, Stifter CA. Negative emotionality and cortisol during adolescent pregnancy and its effects on infant health and autonomic nervous system reactivity. Developmental Psychobiology. 1997; 33:163-174. [PubMed: 9742411]

Radloff LS. The CES-D scale: A self-report depression scale for research in the general population. Applied Psychological Measurement. 1977; 1:385-401.

Ramos, MA.; Diaz, MA.; Muñoz, RF.; Urizar, GG. The Mothers and Babies Course: Relaxation Methods for Managing Stress. 2002. Retrieved February 18, 2006, from http://www.medschool.ucsf.edu/latino/pdf/Relaxation/RelaxationEN.pdf

Robins LN, Helzer JE, Croughan J, Ratcliff KS. National Institute of Mental Health Diagnostic Interview Schedule. Its history, characteristics, and validity. Archives of General Psychiatry. 1981; 38:381-389. [PubMed: 6260053] 
Rosmond R, Björntorp P. The hypothalamic-pituitary-adrenal axis activity as a predictor of cardiovascular disease, type 2 diabetes and stroke. Journal of Internal Medicine. 2000; 247:188197. [PubMed: 10692081]

Spector, PC.; Goodnight, JH.; Sall, JP.; Sarle, WS. SAS User's Guide: Statistics, Version 5. Cary, NC: SAS Institute; 1985. The GLM procedure; p. 433-506.

Spitzer RL, Williams JBW, Kroenke K, Linzer M, deGruy FV, Hahn SR, Brody D, Johnson JG. Utility of a new procedure for diagnosing mental disorders in primary care: the PRIME-MD 1000 study. JAMA. 1994; 272(22):1749-1756. [PubMed: 7966923]

Tu M, Lupien S, Walker C. Diurnal salivary cortisol levels in postpartum mothers as a function of infant feeding choice and parity. Psychoneuroendocrinology. 2006; 31:812-824. [PubMed: 16716531]

Urizar G, Milazzo M, Muñoz RF, Le H, Delucchi K, Sotelo R. Impact of stress reduction instructions on stress and cortisol levels during pregnancy. Biological Psychology. 2004; 67(3):275-282. [PubMed: 15294386]

Watson D, Clark LA, Tellegen A. Development and validation of brief measures of positive and negative affect: The PANAS scales. Journal of Personality \& Social Psychology. 1988; 54(6): 1063-1070. [PubMed: 3397865]

Wesley Y. Reduce stress: A stress reduction project for pregnant Black women. Journal of Cultural Diversity. 2006; 13(4):208-216. [PubMed: 17338491]

Young EA, Aggen SH, Prescott CA, Kendler KS. Similarity in saliva cortisol measures in monozygotic twins and the influence of part major depression. Biological Psychiatry. 2000; 48:70-74. [PubMed: 10913510]

Zich JM, Attkisson CC, Greenfield TK. Screening for depression in primary care clinics: the CES-D and the BDI. International Journal of Psychiatry in Medicine. 1990; 20(3):259-77. [PubMed: 2265888] 


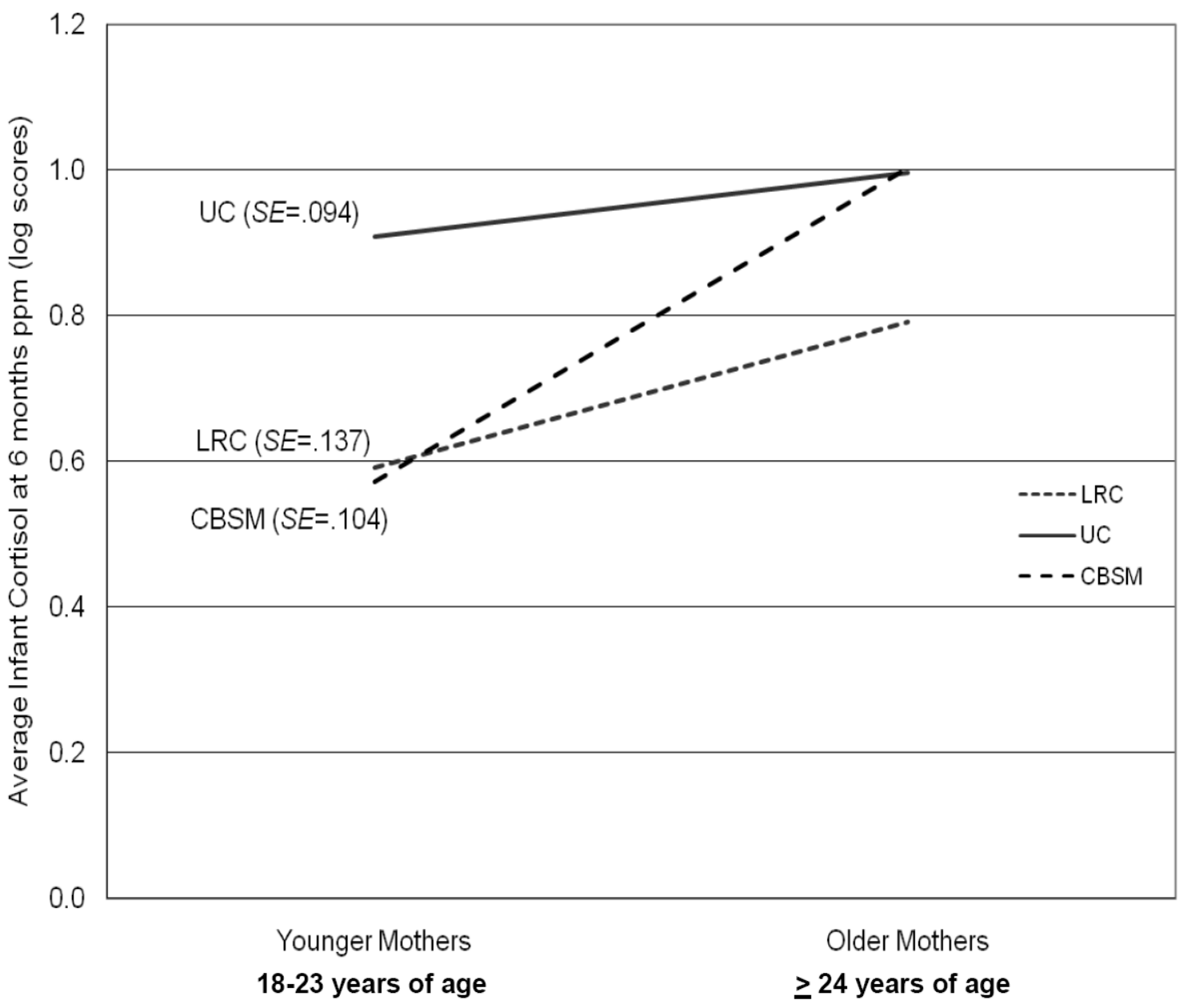

Figure 1.

Descriptive plot illustrating the interaction of group condition by maternal age on infant cortisol at six months postpartum. 


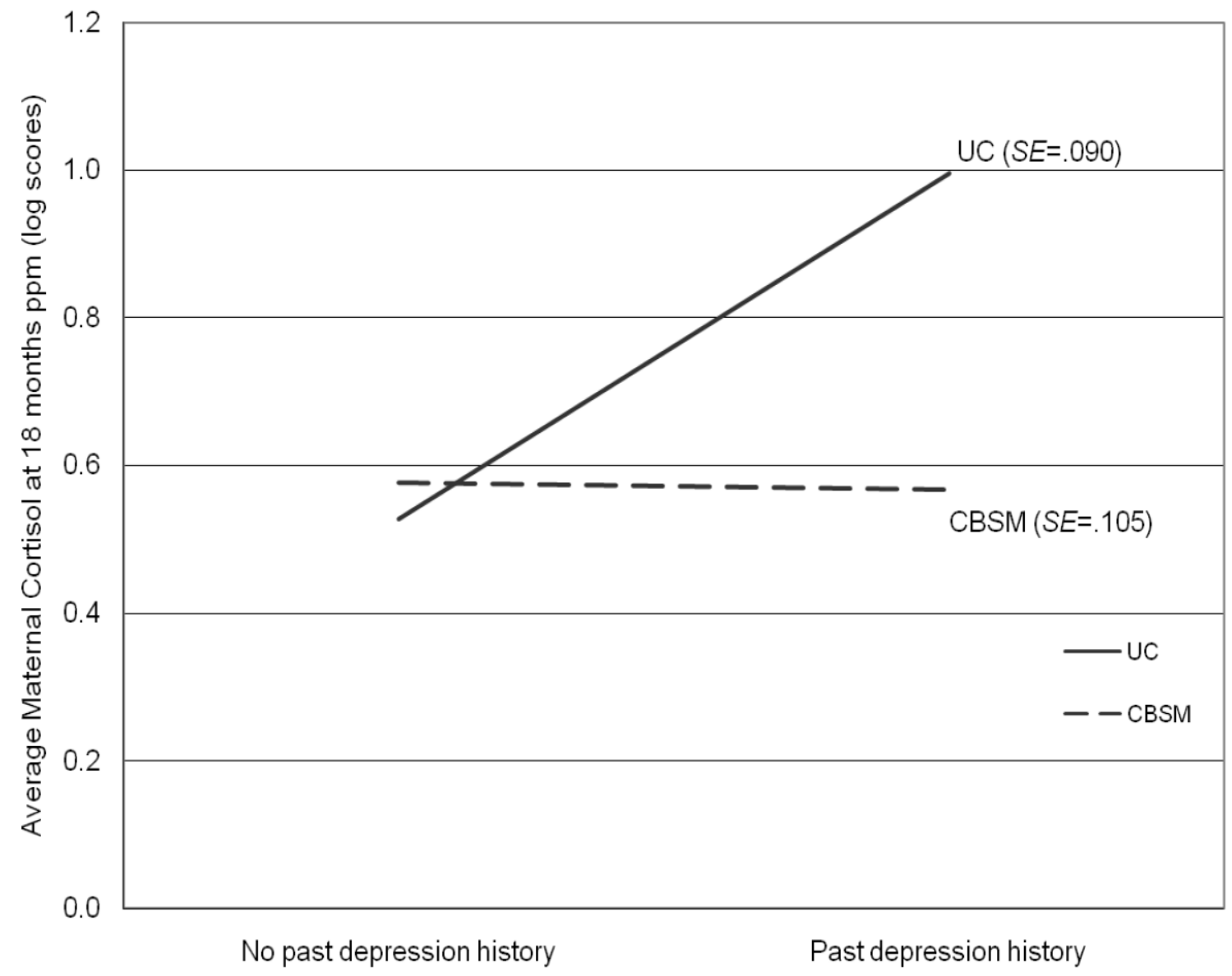

Figure 2.

Descriptive plot illustrating the interaction of group condition by past history of depression on maternal cortisol at 18 months postpartum. 


\section{Table 1}

Sociodemographic characteristics for mothers and their children by group condition.

\begin{tabular}{lccc}
\hline & CBSM $\boldsymbol{n}=\mathbf{2 4}$ & UC $\boldsymbol{n}=\mathbf{3 3}$ & LRC $\boldsymbol{n}=\mathbf{2 9}$ \\
\hline Maternal characteristics & & & \\
Age $[M(S D)]$ & $25.0( \pm 4.7)$ & $25.6( \pm 4.7)$ & $25.6( \pm 4.3)$ \\
Number of weeks pregnant $[M(S D)]$ & $16.4( \pm 4.9)$ & $15.9( \pm 5.0)$ & $16.9( \pm 5.4)$ \\
First pregnancy $(\%)$ & 39.1 & 37.5 & 44.8 \\
Number of live children $[M(S D)]$ & $0.9( \pm 1.1)$ & $1.2( \pm 1.4)$ & $0.8( \pm 0.8)$ \\
Years of education $[M(S D)]$ & $10.9( \pm 3.1)$ & $10.1( \pm 3.0)$ & $10.4( \pm 3.9)$ \\
Annual combined income $<\$ 20,000(\%)$ & 29.2 & 48.4 & 72.4 \\
Married or living with a partner $(\%)$ & 79.2 & 72.7 & 79.3 \\
Primary language spoken at home, Spanish $(\%)$ & 79.2 & 84.8 & 75.9 \\
Country of birth, Mexico/Central America $(\%)$ & 75.0 & 78.8 & 79.3 \\
Age when immigrated to U.S. $[M(S D)]$ & $19.0( \pm 8.2)$ & $19.6( \pm 5.4)$ & $18.8( \pm 7.6)$ \\
Number of prenatal classes attended $[M(S D)]$ & $7.3( \pm 3.4)$ & - & - \\
Number of booster classes attended $[M(S D)]$ & $1.6( \pm 1.0)$ & - & - \\
Child characteristics & & & \\
Child gender, Male (\%) & 52.2 & 61.3 & 51.7 \\
Number of birth complications $[M(S D)]$ & $1.0( \pm 1.2)$ & $0.9( \pm 1.1)$ & $1.4( \pm 1.4)$ \\
Age in months at 18 month timepoint $[M(S D)]$ & $18.71( \pm 2.2)$ & $19.95( \pm 6.1)$ & - \\
Child's primary language, Spanish $(\%)$ & 69.6 & 65.0 & - \\
\hline
\end{tabular}

Note. Pearson's $\chi^{2}$ and ANOVAs were conducted to examine for between group differences among categorical and continuous variables, respectively.

* $p<.05$;

** $p<.01 ;$

$* * *$

$p<.001$ 
Table 2

Means and standard deviations of salivary cortisol, perceived stress, and maternal mood levels during pregnancy, by group condition.

\begin{tabular}{|c|c|c|c|}
\hline & $\operatorname{CBSM} n=24$ & $\mathrm{UC} n=33$ & LRC $n=29$ \\
\hline & $M(S D)$ & $M(S D)$ & $M(S D)$ \\
\hline \multicolumn{4}{|l|}{ Prepartum salivary cortisol } \\
\hline \multicolumn{4}{|l|}{ Cortisol (log scores) } \\
\hline Morning cortisol & $0.89( \pm 0.26)^{* *}$ & $1.02( \pm 0.22)$ & $1.11( \pm 0.23)$ \\
\hline Evening cortisol & $0.35( \pm 0.26)^{*}$ & $0.48( \pm 0.47)$ & $0.62( \pm 0.40)$ \\
\hline Average cortisol & $0.62( \pm 0.22)^{* *}$ & $0.75( \pm 0.27)$ & $0.87( \pm 0.24)$ \\
\hline Cortisol slope & $0.55( \pm 0.29)$ & $0.53( \pm 0.51)$ & $0.49( \pm 0.44)$ \\
\hline \multicolumn{4}{|l|}{ Collection time [hour:minutes (minutes)] } \\
\hline Morning cortisol collection time & $9: 02( \pm 1: 17)^{*}$ & $8: 11( \pm 1: 06)$ & $8: 47( \pm 0: 54)$ \\
\hline Evening cortisol collection time & 8:08 ( $\pm 0: 29)$ & $8: 07( \pm 0: 21)$ & $8: 11( \pm 0: 22)$ \\
\hline \multicolumn{4}{|l|}{ Prepartum stress and mood measures } \\
\hline \multicolumn{4}{|l|}{ Stress rating } \\
\hline Morning stress rating & $23.0( \pm 24.9)$ & $29.5( \pm 22.8)$ & $23.9( \pm 22.7)$ \\
\hline Evening stress rating & $30.5( \pm 24.0)$ & $32.0( \pm 21.8)$ & $21.4( \pm 17.5)$ \\
\hline Average stress rating & $26.3( \pm 19.8)$ & $30.6( \pm 20.1)$ & $23.4( \pm 19.6)$ \\
\hline \multicolumn{4}{|l|}{ Depression measures } \\
\hline Past History of Major Depression (\%) & 33.3 & 66.7 & $0.0^{* * *}$ \\
\hline CES-D & $20.6( \pm 7.9)$ & $23.7( \pm 12.3)$ & $9.4( \pm 3.6)^{* * *}$ \\
\hline \multicolumn{4}{|l|}{ Affect } \\
\hline Positive affect & $2.5( \pm 0.8)$ & $2.4( \pm 0.9)$ & $3.0( \pm 1.0)$ \\
\hline Negative affect & $1.6( \pm 0.5)$ & $1.6( \pm 0.6)$ & $1.5( \pm 0.5)$ \\
\hline
\end{tabular}

Note. Pearson's $\chi^{2}$ and ANOVAs were conducted to examine for between group differences among categorical and continuous variables, respectively.

* $p<.05$;

*** $p<.01$

$* * * 0.001$ 


\section{Table 3}

Means and standard deviations of salivary cortisol, perceived stress, and maternal mood levels at 6 months postpartum, by group condition.

\begin{tabular}{|c|c|c|c|}
\hline & $\operatorname{CBSM} n=24$ & UC $n=29$ & LRC $n=22$ \\
\hline & $M(S D)$ & $M(S D)$ & $M(S D)$ \\
\hline \multicolumn{4}{|l|}{ 6-month postpartum salivary cortisol } \\
\hline \multicolumn{4}{|l|}{ Maternal cortisol (log scores) } \\
\hline Morning cortisol & $0.93( \pm 0.23)$ & $0.81( \pm 0.48)$ & $0.83( \pm 0.40)$ \\
\hline Evening cortisol & $0.30( \pm 0.65)$ & $0.13( \pm 0.50)$ & $0.10( \pm 0.48)$ \\
\hline Average cortisol & $0.62( \pm 0.40)$ & $0.47( \pm 0.39)$ & $0.43( \pm 0.35)$ \\
\hline Cortisol slope & $0.62( \pm 0.56)$ & $0.68( \pm 0.58)$ & $0.76( \pm 0.46)$ \\
\hline \multicolumn{4}{|l|}{ Infant cortisol (log scores) } \\
\hline Morning cortisol & $1.03( \pm 0.49)$ & $1.16( \pm 0.33)$ & $0.96( \pm 0.42)$ \\
\hline Evening cortisol & $0.63( \pm 0.52)$ & $0.76( \pm 0.52)$ & $0.60( \pm 0.49)$ \\
\hline Average cortisol ${ }^{\dagger}$ & $0.84( \pm 0.48)$ & $0.96( \pm 0.39)^{*}$ & $0.75( \pm 0.31)$ \\
\hline Cortisol slope & $0.41( \pm 0.34)$ & $0.40( \pm 0.41)$ & $0.46( \pm 0.55)$ \\
\hline \multicolumn{4}{|l|}{ Collection time [hour:minutes (minutes)] } \\
\hline Mother morning cortisol collection time & $8: 21( \pm 1: 05)$ & $8: 17( \pm 0: 52)$ & $8: 18( \pm 0: 55)$ \\
\hline Mother evening cortisol collection time & $8: 15( \pm 0: 31)$ & $8: 10( \pm 0: 24)$ & $8: 08( \pm 0: 45)$ \\
\hline Infant morning cortisol collection time & $8: 31( \pm 1: 06)$ & $8: 21( \pm 0: 51)$ & $8: 02( \pm 1: 43)$ \\
\hline Infant evening cortisol collection time & $8: 18( \pm 0: 31)$ & $8: 10( \pm 0: 24)$ & $8: 04( \pm 0: 46)$ \\
\hline \multicolumn{4}{|l|}{ 6-month postpartum stress and mood measures } \\
\hline \multicolumn{4}{|l|}{ Maternal stress rating } \\
\hline Morning stress rating & $39.0( \pm 19.7)^{*}$ & $28.5( \pm 21.4)$ & $21.0( \pm 20.3)$ \\
\hline Evening stress rating & $45.8( \pm 29.5)^{*}$ & $33.5( \pm 25.0)$ & $26.3( \pm 21.9)$ \\
\hline Average stress rating & $41.4( \pm 21.1)^{*}$ & $30.9( \pm 22.3)$ & $23.0( \pm 20.1)$ \\
\hline \multicolumn{4}{|l|}{ Depression } \\
\hline CES-D & $19.6( \pm 12.9)$ & $17.0( \pm 12.9)$ & $11.3( \pm 7.8)$ \\
\hline \multicolumn{4}{|l|}{ Affect } \\
\hline Positive affect & $1.6( \pm 0.7)$ & $1.8( \pm 0.7)$ & $2.4( \pm 1.1)^{*}$ \\
\hline Negative affect & $2.5( \pm 0.8)$ & $2.4( \pm 0.8)$ & $2.3( \pm 1.1)$ \\
\hline
\end{tabular}

Note. ANOVAs were conducted for all between group analyses

* $p<.05$;

** $p<.01$

$* * * \quad p<.001$

${ }^{\dagger}$ Group means were significant after controlling for maternal cortisol and maternal age $(p<.001)$ 
Table 4

Means and standard deviations of salivary cortisol, perceived stress, and maternal mood levels at 18 months postpartum, by group condition.

\begin{tabular}{|c|c|c|}
\hline & $\operatorname{CBSM} n=24$ & $\mathrm{UC} n=22$ \\
\hline & $M(S D)$ & $M(S D)$ \\
\hline \multicolumn{3}{|l|}{ 18-month postpartum salivary cortisol } \\
\hline \multicolumn{3}{|l|}{ Maternal cortisol (log scores) } \\
\hline Morning cortisol & $0.98( \pm 0.32)$ & $1.23( \pm 0.58)$ \\
\hline Evening cortisol & $0.20( \pm 0.52)$ & $0.40( \pm 0.45)$ \\
\hline Average cortisol ${ }^{\dagger}$ & $0.60( \pm 0.36)$ & $0.81( \pm 0.43)^{*}$ \\
\hline Cortisol slope & $0.79( \pm 0.47)$ & $0.83( \pm 0.56)$ \\
\hline \multicolumn{3}{|l|}{ Child cortisol (log scores) } \\
\hline Morning cortisol & $1.00( \pm 0.49)$ & $1.18( \pm 0.31)$ \\
\hline Evening cortisol & $0.53( \pm 0.61)$ & $0.58( \pm 0.72)$ \\
\hline Average cortisol & $0.76( \pm 0.50)$ & $0.82( \pm 0.61)$ \\
\hline Cortisol slope & $0.45( \pm 0.48)$ & $0.50( \pm 0.54)$ \\
\hline \multicolumn{3}{|l|}{ Collection time [hour:minutes (minutes)] } \\
\hline Mother morning cortisol collection time & $8: 39( \pm 1: 05)$ & $8: 21( \pm 1: 05)$ \\
\hline Mother evening cortisol collection time & $8: 15( \pm 0: 35)$ & $8: 11( \pm 0: 38)$ \\
\hline Child morning cortisol collection time & $8: 58( \pm 0: 59)$ & $8: 22( \pm 1: 03)$ \\
\hline Child evening cortisol collection time & $8: 16( \pm 0: 21)$ & $8: 12( \pm 0: 38)$ \\
\hline \multicolumn{3}{|l|}{ 18-month postpartum stress and mood measures } \\
\hline \multicolumn{3}{|l|}{ Maternal stress rating } \\
\hline Morning stress rating & $32.2( \pm 19.6)$ & $33.0( \pm 18.7)$ \\
\hline Evening stress rating & $46.3( \pm 21.1)$ & $41.1( \pm 21.1)$ \\
\hline Average stress rating & $39.7( \pm 17.7)$ & $37.5( \pm 18.1)$ \\
\hline \multicolumn{3}{|l|}{ Child stress rating } \\
\hline Morning stress rating & $20.5( \pm 20.1)$ & $23.5( \pm 19.0)$ \\
\hline Evening stress rating & $40.0( \pm 27.1)$ & $26.8( \pm 22.4)$ \\
\hline Average stress rating & $30.3( \pm 20.7)$ & $25.3( \pm 18.2)$ \\
\hline \multicolumn{3}{|l|}{ Depression } \\
\hline CES-D & $15.4( \pm 10.5)$ & $15.8( \pm 10.5)$ \\
\hline \multicolumn{3}{|l|}{ Affect } \\
\hline Positive affect & $2.7( \pm 0.6)$ & $2.5( \pm 0.8)$ \\
\hline Negative affect & $1.7( \pm 0.7)$ & $1.7( \pm 0.8)$ \\
\hline
\end{tabular}

Note. Independent samples $t$ tests were conducted for all between group analyses

$p<.05$

$* *$

$p<.01$;

**** $p<.001$

${ }^{\dagger}$ Group means were significant after adjusting for past history of depression and annual family income $(p<.01)$

Psychoneuroendocrinology. Author manuscript; available in PMC 2012 November 01. 